Pacific

Journal of

Mathematics

\title{
WEAKLY REGULAR EMBEDDINGS OF STEIN SPACES WITH
} ISOLATED SINGULARITIES

\author{
JASNa PRezelu
}




\title{
WEAKLY REGULAR EMBEDDINGS OF STEIN SPACES WITH ISOLATED SINGULARITIES
}

\author{
JASNA PREZELJ
}

\begin{abstract}
We show that any $n$-dimensional Stein space $X$ with isolated singular points admits a proper holomorphic injective map $X \rightarrow \mathbb{C}^{2 n}$ which is regular on $\operatorname{Reg}(X)$. The proof is based on the fact that the Whitney cones $C_{5}(x, X)$ are at most $2 n$-dimensional, which means that there exists a neighborhood of $x$ in $X$ having a weakly regular embedding into $\mathbb{C}^{2 n}$. The homotopic principle then enables us to obtain a weakly regular embedding of $X$ into $\mathbb{C}^{2 n}$.
\end{abstract}

\section{Introduction}

The motivation for this paper was the following question: Let $M$ be a smooth, compact, strongly pseudoconvex, integrable CR-manifold of dimension $2 n-1 \geq 5$ and of CR-dimension $n-1 \geq 2$. Find the smallest integer $N=N(n)$ such that $M$ admits a CR embedding into $\mathbb{C}^{N}$. By the results of Rossi [1965] and Ohsawa [1984a; 1984b], there exists a pure $n$-dimensional Stein space $X$ with isolated singular points and a relatively compact domain $D \subset X$ such that $\partial D=M$ and $\partial D \cap \operatorname{Sing}(X)=\varnothing$. This leads to the following problem: Let $X$ be an $n$-dimensional Stein space with isolated singular points. Find the smallest integer $N$ such that there exists a proper holomorphic injective map $f: X \rightarrow \mathbb{C}^{N}$ which is regular on $\operatorname{Reg}(X)$. It turns out that the dimension $N$ can be expressed in terms of the Whitney cones $C_{5}$ (for the definition see Section 2 or [Chirka 1989]).

Theorem 1.1. Let $X$ be an $n$-dimensional Stein space with isolated singular points. Let $N(X)=\max \left\{[n / 2]+n+1,3, \max \left\{\operatorname{dim} C_{5}(x, X): x \in X\right\}\right\}$. Then there exists a proper holomorphic injective map $f: X \rightarrow \mathbb{C}^{N(X)}$, which is regular on $\operatorname{Reg}(X)$.

Remark 1.2. Since we are not interested in regularity at singular points we may (and will), with no loss of generality, assume that the space is reduced. By [Acquistapace et al. 1975] there is a proper holomorphic injective map $f: X \rightarrow \mathbb{C}^{N}$,

MSC2000: 32C15, 32C22, 32E10, 32H02.

Keywords: Stein space, holomorphic map, weakly regular embedding, homotopic principle,

Whitney cone.

The author was supported by the Ministry of Education, Science and Sports of the Republic of Slovenia. 
where $N \geq 2 n+1$, which is regular on $\operatorname{Reg}(X)$. The dimension $N(X)$ from Theorem 1.1, however, is at most $2 n$, because $\operatorname{dim} C_{5}(x, X) \leq 2 n$ for all $n$-dimensional Stein spaces $X$ and all $x \in X$. If $X$ is a Stein manifold, it was proved by Schürman that $N(X)=[n / 2]+n+1$ for $n>1$.

Remark 1.3. In the case of normal Stein spaces any two weakly regular holomorphic embeddings are biholomorphically equivalent. Let us mention that this result does not necessarily give a minimal $N$ for CR-embedding.

The paper is organized as follows: the second section contains the definition and some properties of Whitney cones and the third section consists of the proof of the main theorem.

Definitions and notation. For $y \in \mathbb{C}^{n}$ let $|y|:=\sup \left\{\left|y_{i}\right|: 1 \leq i \leq n\right\}$ denote the sup norm and $\|y\|$ the euclidean norm. By $B_{n}(r)$ we denote the ball in $\mathbb{C}^{n}$ with radius $r$ and center 0 .

Let $X$ be a complex space, $K \subset X$ a compact subset, and $f: X \rightarrow \mathbb{C}^{n}$ a continuous map. We will use the notation $|f|_{K}:=\max \{|f(x)|: x \in K\}$ and $\|f\|_{K}:=\max \{\|f(x)\|: x \in K\}$. By $\mathcal{O}(X)$ we denote the space of all holomorphic functions on a complex space $X$ equipped with the standard topology of uniform convergence on compact sets. For an analytic set $Y \subset X$ let $\Gamma(X, \mathscr{g}(Y))$ denote the space of holomorphic functions on $X$ which vanish on $Y$. By $T X$ we denote the complex tangent space of $X$ and by $T_{x} X$ the complex tangent space of $X$ at the point $x$.

A holomorphic map $f: X \rightarrow Y$ is almost proper if for each compact set $K \subset Y$ the connected components of $f^{-1}(K)$ are compact. A stratification of a complex space $X$ is a finite descending chain of analytic sets $A_{m}:=X \supset A_{m-1} \supset \cdots \supset A_{0}$ such that $A_{i} \backslash A_{i-1}$ is a complex manifold, for $i=1, \ldots, m$.

\section{Some properties of tangent cones}

The Whitney tangent cones $C_{3}, C_{4}$ and $C_{5}$ play an important role in our work.

Definition 2.1. Let $X \subset \mathbb{C}^{m}$ be an analytic set, $x \in X$. Then let

$C_{3}(x, X):=\left\{v \in \mathbb{C}^{m}:\right.$ there exists a sequence $x_{j} \in X$ such that $x_{j} \rightarrow x$, and a sequence $\lambda_{j} \in \mathbb{C}$ such that $\left.\lambda_{j}\left(x_{j}-x\right) \rightarrow v\right\}$,

$C_{4}(x, X):=\left\{v \in \mathbb{C}^{m}:\right.$ there exists a sequence $z_{j} \in \operatorname{Reg}(X)$ such that $z_{j} \rightarrow x$, and a sequence $v_{j} \in T_{z_{j}} X$ such that $v_{j} \rightarrow v$ \}, $C_{5}(x, X):=\left\{v \in \mathbb{C}^{m}:\right.$ there exist sequences $z_{j}, w_{j} \in X$ with $z_{j}, w_{j} \rightarrow x$, and a sequence $\lambda_{j} \in \mathbb{C}$ such that $\left.\lambda_{j}\left(z_{j}-w_{j}\right) \rightarrow v\right\}$.

Further, set $C_{3}(X):=\left\{(x, v): x \in X, v \in C_{3}(x, X)\right\}$ and define $C_{4}(X)$ and $C_{5}(X)$ similarly. Clearly these are three subsets of $T X$. Using the fact that every analytic 
set is locally biholomorphic to an analytic set in some $\mathbb{C}^{m}$, we can extend the above definition of cones to an arbitrary complex space $X$. A more detailed discussion on this subject can be found in [Chirka 1989; Stutz 1972]. We state some simple properties of Whitney cones [Chirka 1989, Sections 8.4, 9.1 and 9.2]. If $n=$ $\operatorname{dim}(x, X)$, then:

(i) The cones $C_{4}(x, X)$ and $C_{5}(x, X)$ are biholomorphically invariant, projective algebraic sets with $n \leq \operatorname{dim} C_{i}(x, X) \leq 2 n$, and the cone $C_{3}(x, X)$ is an $n$ dimensional algebraic set.

(ii) $C_{3}(x, X) \subset C_{4}(x, X) \subset C_{5}(x, X)$.

(iii) $C_{4}(X)$ is the closure of $\left.T X\right|_{\operatorname{Reg}(X)}$.

(iv) If $x \in \operatorname{Reg}(X)$ then $\operatorname{dim} C_{4}(x, X)=\operatorname{dim} C_{5}(x, X)=n$.

(v) If $\operatorname{dim} C_{5}(x, X)=n$, then $x \in \operatorname{Reg}(X)$.

Example 2.2. Let $X=\left(\mathbb{C}^{n} \times 0\right) \cup\left(0 \times \mathbb{C}^{n}\right) \subset \mathbb{C}^{2 n}$. Then $C_{3}(0, X)=C_{4}(0, X)=X$ and $C_{5}(0, X)=\mathbb{C}^{2 n}$.

Proposition 2.3 [Chirka 1989, Section 8.4]. Let $X \subset \mathbb{C}^{m}$ be an analytic set containing 0 , let $L=\mathbb{C}^{m-k} \times 0 \subset \mathbb{C}^{m}$, and suppose that $C_{3}(0, X) \cap L=\{0\}$. Then there exists an open set $U \subset \mathbb{C}^{m}$ containing 0 , such that the orthogonal projection $\pi_{L}: U \cap X \rightarrow \mathbb{C}^{k}$ is proper.

Remark 2.4. The condition $C_{3}(0, X) \cap L=\{0\}$ implies that the neighborhood of 0 lies in some cone. The condition is fulfilled for almost every $(m-k)$-dimensional linear subspace $L \subset \mathbb{C}^{m}$. Clearly, the projection along any $L$ with $\operatorname{dim} L \leq m-n$ and $C_{3}(0, X) \cap L=\{0\}$ is also proper.

Proposition 2.5 [Chirka 1989, Section 9.4]. Let $X \subset \mathbb{C}^{m}$ be a pure $n$-dimensional analytic set containing 0 , let $L=\mathbb{C}^{m-n} \times 0 \subset \mathbb{C}^{m}$, and let $\pi_{L}: U \cap X \rightarrow \mathbb{C}^{n}$ be the orthogonal projection. If $C_{4}(0, X) \cap L=\{0\}$ then there exists an open set $U \subset \mathbb{C}^{m}$ containing 0 , such that $\operatorname{br}\left(\pi_{L}, X \cap U\right)=(X \backslash \operatorname{Reg}(X)) \cap U$.

Remark 2.6. In the case of a general $n$-dimensional analytic set such projection is of course not a cover; it is, however, proper (because $\left.C_{3}(0, X) \subset C_{4}(0, X)\right)$ and regular on $\operatorname{Reg}(X) \cap U$.

Corollary 2.7. Let $X$ be a complex space, let $x \in X$ and suppose $\operatorname{dim} C_{4}(x, X)=k$. Then there exists an open neighborhood $U$ of $x$, and a proper holomorphic map $f: U \rightarrow \mathbb{C}^{k}$ which is regular on $\operatorname{Reg}(X) \cap U$. Every holomorphic map $f: X \rightarrow \mathbb{C}^{k}$ with $\operatorname{Ker} D f(x) \cap C_{4}(x, X)=\{0\}$ is regular on $\operatorname{Reg}(X) \cap U$ for a suitable open neighborhood $U$ of $x$. 
Proof. We may assume that $X \subset \mathbb{C}^{m}$, since the statement is local. For the first part, notice that the condition $\operatorname{dim} C_{4}(x, X)=k$ implies the existence of an $(m-k)$ dimensional linear subspace $L$ with $L \cap C_{4}(x, X)=\{0\}$. The rest follows from Proposition 2.5 and the remark below.

As for the second part, if the statement is false, there exist sequences $x_{j} \in$ $\operatorname{Reg}(X)$ with $x_{j} \rightarrow x$, and $v_{j} \in T_{x_{j}} X$ with $\left\|v_{j}\right\|=1$, such that

$$
D f\left(x_{j}\right)\left(v_{j}\right)=0 .
$$

By passing to a subsequence we may assume that $v_{j} \rightarrow v$. But $v$ is in $C_{4}(x, X)$ by definition; therefore $D f(x)(v)=0$, which is a contradiction.

Proposition 2.8 [Chirka 1989, Section 9.4]. Let $X \subset \mathbb{C}^{m}$ be a pure $n$-dimensional analytic set containing 0 , and let $L=\mathbb{C}^{m-n-1} \times 0 \subset \mathbb{C}^{m}$. If $C_{5}(0, X) \cap L=\{0\}$ then there exists an open set $U \subset \mathbb{C}^{m}$ such that the orthogonal projection $\pi_{L}: U \rightarrow$ $\mathbb{C}^{n+1}$ is an almost one-sheeted cover over some analytic subset of $\mathbb{C}^{n+1}$, that is, a homeomorphism of $X \cap U$ onto some hypersurface in $U \cap \mathbb{C}^{n+1}$.

Remark 2.9. As before in the case of a general $n$-dimensional analytic set such a projection is not a cover; it is proper (because $C_{3}(0, X) \subset C_{5}(0, X)$ ), regular on $\operatorname{Reg}(X) \cap U$ and injective.

Corollary 2.10. Let $X$ be a complex space, let $x \in X$, and let $\operatorname{dim} C_{5}(x, X)=k$. Then there exists an open neighborhood $U$ of $x$, and a proper, injective holomorphic map $f: U \rightarrow \mathbb{C}^{k}$, which is regular on $\operatorname{Reg}(X) \cap U$. Every holomorphic map $f: X \rightarrow \mathbb{C}^{k}$ satisfying $\operatorname{Ker} D f(x) \cap C_{5}(x, X)=\{0\}$ is injective, proper and regular on $\operatorname{Reg}(X) \cap U$ for some neighborhood $U$ of $x$.

Proof. The first part follows by a similar argument to that used in Corollary 2.7. We may assume that $X \subset \mathbb{C}^{m}$. Because $C_{4}(x, X) \subset C_{5}(x, X)$, the regularity of the map on $\operatorname{Reg}(X) \cap U$, for some small neighborhood $U$ of $x$, follows from Corollary 2.7. If the map were not injective in any neighborhood of $x$ then there would exist sequences $x_{j}, y_{j} \in X$ with $x_{j}, y_{j} \rightarrow x$ and $x_{j} \neq y_{j}$, such that $f\left(x_{j}\right)-f\left(y_{j}\right)=0$. The Taylor series expansion gives us

$$
f\left(x_{j}\right)-f\left(y_{j}\right)=D f\left(x_{j}\right)\left(x_{j}-y_{j}\right)+o\left(\left|x_{j}-y_{j}\right|\right)=0,
$$

which means that

$$
D f\left(x_{j}\right)\left(\frac{x_{j}-y_{j}}{\left|x_{j}-y_{j}\right|}\right) \rightarrow 0 .
$$

By passing to a subsequence we may assume that $\left(x_{j}-y_{j}\right) /\left|x_{j}-y_{j}\right| \rightarrow v$, which lies in $C_{5}(x, X)$. But then $D f(x)(v)=0$, which contradicts the assumption.

Definition 2.11. Let $X$ be a complex space, let $x \in X$, and let $f: X \rightarrow \mathbb{C}^{m}$ be a holomorphic map. The map $f$ is weakly regular at $x$ if $C_{5}(x, X) \cap \operatorname{Ker} D f(x)=\{0\}$ and weakly regular if $C_{5}(X) \cap \operatorname{Ker} D f=0$, where 0 is the zero section in $T X$. 
On a complex manifold the notions of regular and weakly regular coincide. One of the key features of weakly regular maps that will be used in the sequel is local injectivity. Let us state two more lemmas describing properties of injective weakly regular maps.

Lemma 2.12. Let $X$ be a complex space, let $K \subset X$ be a compact set and let $f: X \rightarrow \mathbb{C}^{m}$ be a holomorphic map which is weakly regular and injective on $K$. Then there exists an open neighborhood $U \subset X$ of $K$ such that $f$ is injective and weakly regular on $U$.

Proof. Weak regularity is obviously an open condition. Assume that the map is not injective. Then there are sequences $x_{j}, y_{j} \in X$, with $x_{j} \neq y_{j}$, such that $x_{j} \rightarrow x \in K$, $y_{j} \rightarrow y \in K$ and $f\left(x_{j}\right)=f\left(y_{j}\right)$. Injectivity of $f$ on $K$ implies that $x=y$ and since $f$ is weakly regular on $K$ it is injective in a neighborhood of $x$, which contradicts the existence of the sequences $x_{j}$ and $y_{j}$.

Lemma 2.13. Let $X$ be a complex space, let $K \subset X$ be a compact set, and let $f: X \rightarrow \mathbb{C}^{m}$ be a holomorphic map which is weakly regular and injective on $K$. Then there exists an $\varepsilon>0$ such that any holomorphic map $g: X \rightarrow \mathbb{C}^{m}$ satisfying $|g-f|_{K}<\varepsilon$ is injective and weakly regular on $K$.

Proof. Every map $g$ close enough to $f$ on $K$ is weakly regular on $K$ and therefore locally injective. The next step is to prove a local result:

Claim. Take $x \in X$ and assume that the map $f$ is weakly regular (and therefore injective) in a small compact neighborhood $U$ of $x$. Then there exists an $\varepsilon>0$ such that if $|g-f|_{U}<\varepsilon$, then $g$ is injective and weakly regular on $U$.

Proof of the claim. Note that any map close to $f$ is weakly regular at $x$ and therefore injective in some neighborhood of $x$. We need to prove that the map is injective on $U$. Assume the converse. Then there exists a sequence $\varepsilon_{j} \rightarrow 0$, a sequence $g_{j}: X \rightarrow \mathbb{C}^{m}$, and sequences $x_{j}, y_{j} \in U$, such that $\left|g_{j}-f\right|<\varepsilon_{j}$ and such that $g_{j}\left(x_{j}\right)-g_{j}\left(y_{j}\right)=0$. We may assume that $x_{j} \rightarrow x$ and $y_{j} \rightarrow y$, and also that $\left(x_{j}-y_{j}\right) /\left|x_{j}-y_{j}\right| \rightarrow v$. Injectivity of $f$ implies $x=y$. The Taylor series expansion gives

$$
D g_{j}\left(x_{j}\right)\left(x_{j}-y_{j}\right)=o_{j}\left(\left|x_{j}-y_{j}\right|\right) \text {. }
$$

Because of the Cauchy estimates there is $o\left(\left|x_{j}-y_{j}\right|\right)$ such that

$$
\left|o_{j}\left(\left|x_{j}-y_{j}\right|\right)\right|<\left|o\left(\left|x_{j}-y_{j}\right|\right)\right|
$$

for all $j$. Dividing the above equation by $\left|x_{j}-y_{j}\right|$ and passing to the limit we get $D f(x)(v)=0$ which contradicts the fact that $f$ is weakly regular.

We have proved that there exists an open neighborhood $V$ of the diagonal $\Delta \subset$ $K \times K$ such that if $g$ is close enough to $f$, the map $g(x)-g(y): X \times X \rightarrow \mathbb{C}^{m}$ 
will have no zeroes in $V$ except the diagonal $\Delta$. Injectivity of $f$ implies that $\min \{|f(x)-f(y)|:(x, y) \in K \times K \backslash V\}>0$. The same holds for each map $g$ close enough to $f$ on $K$, which means that any such $g$ is injective on $K$.

\section{Proof of the main theorem}

Let $X$ be a $n$-dimensional Stein space with $S=\left\{s_{j}\right\}=X \backslash \operatorname{Reg}(X)$ discrete and let $N=N(X)=\max \left\{[n / 2]+n+1,3, \max \left\{\operatorname{dim} C_{5}(x, X): x \in X\right\}\right\}$. We seek a proper, holomorphic, weakly regular, injective map $F=(H, G): X \rightarrow \mathbb{C}^{n} \times \mathbb{C}^{N-n}$. We first construct an almost proper holomorphic map $H: X \rightarrow \mathbb{C}^{n}$ having certain additional properties (a generic almost proper map) and then construct the map $G: X \rightarrow \mathbb{C}^{N-n}$ such that $F=(H, G)$ has the desired properties.

By the definition of $N$ there exist injective weakly regular holomorphic maps $\Phi_{j}: U_{j} \rightarrow \mathbb{C}^{N}$ defined on small neighborhoods $U_{j}$ of $s_{j}$. For simplicity let us assume that $\Phi_{j, N}\left(s_{j}\right)=j$. Since $X$ is Stein there is a holomorphic map $\Phi: X \rightarrow \mathbb{C}^{N}$ which coincides with the $\Phi_{j}$ to second order on $S$. Define $\varphi=\left(\Phi_{1}, \ldots, \Phi_{n}\right)$. The following theorem gives a generic almost proper map $H$ which coincides with $\varphi$ on $S$ to second order.

Proposition 3.1 (Generic almost proper maps) [Schürmann 1997; Prezelj 2003]. Let $X$ be a $n$-dimensional Stein space, let $Y \subset X$ be a discrete set, let $\operatorname{Sing}(X) \subset Y$, let $\varphi: X \rightarrow \mathbb{C}^{n}$ be a holomorphic map, and let $q^{\prime}=\left[\frac{n+1}{2}\right]$. For each $y \in Y$ let a number $m_{y} \in \mathbb{N}$ be given. The set of all almost proper holomorphic maps $H: X \rightarrow \mathbb{C}^{n}$ satisfying

(i) $(H-\varphi)_{y} \in \mathscr{G}(Y)^{m_{j}}$ for each $y \in Y$, and

(ii) $\operatorname{dim}\left\{x \in X \backslash Y: \operatorname{rank}_{x} H \leq n-i\right\}<2\left(q^{\prime}-i+1\right)$, for $i=1, \ldots, n$

is residual in the set $G$ of all holomorphic maps $G$ satisfying $(G-\varphi)_{y} \in \mathscr{L}(Y)^{m_{j}}$ for each $y \in Y$.

Remark 3.2. The first theorem of this sort was proved in [Schürmann 1997, Proposition 4.1]; see [Prezelj 2003, Proposition 2.4] for modifications. In our case $m_{j}=2$ for each $j$. The maps $H$ and $\varphi$ will be fixed through the rest of the section.

The construction of the map $G$ requires more work. We follow the proof of the embedding theorem in [Schürmann 1992]. The full proof is quite long and complicated, so we will only explain how to modify the theorems so that they hold for weakly regular maps. The main tool in the proof is the h-principle:

Definition 3.3 [Gromov 1989]. Let $Z$ and $X$ be complex spaces, let $h: Z \rightarrow X$ be a surjective submersion, and let $U \subset X$ be an open set. Then $h$ admits a spray over $U$ if, for some $m \in \mathbb{N}$, there exists a holomorphic map $s: h^{-1}(U) \times \mathbb{C}^{m} \rightarrow h^{-1}(U)$ such that 
(i) $s(z, 0)=z$ for each $z \in h^{-1}(U)$,

(ii) $s\left(z, \mathbb{C}^{m}\right) \subset h^{-1}(h(z))$ for each $z \in h^{-1}(U)$, and

(iii) $\left.(\partial / \partial t) s(z, t)\right|_{t=0}: \mathbb{C}^{m} \rightarrow \operatorname{Ker} D_{z} h$ is surjective.

Theorem 3.4 (The h-principle for Stein spaces) [Gromov 1989; Forstnerič and Prezelj 2001]. Let $X$ be a Stein space, $Z$ a complex space and $h: Z \rightarrow X a$ holomorphic submersion (with constant corank) onto $X$. Assume that each $x \in X$ has a neighborhood $U \subset X$ such that h admits a spray over $U$. Let $d$ be a metric on $Z$ compatible with the complex space topology. Then:

(i) Each continuous section $f_{0}: X \rightarrow Z$ can be deformed to a holomorphic section $f_{1}: X \rightarrow Z$ through a continuous one-parameter family of continuous sections (a homotopy) $f_{t}: X \rightarrow Z$, for $t \in[0,1]$.

(ii) If $K \subset X$ is a compact holomorphically convex set and the initial section $f_{0}$ is holomorphic in a neighborhood of $K$, then for each $\varepsilon>0$ there exists a homotopy $f_{t}: X \rightarrow Z$, for $t \in[0,1]$, such that $d\left(f_{t}(x), f_{0}(x)\right)<\varepsilon$ for each $x \in K$ and $t \in[0,1]$, each $f_{t}$ is holomorphic in a neighborhood of $K$ and $f_{1}$ is holomorphic on $X$. In this case it suffices to assume that the submersion $h: Z \rightarrow X$ has a spray over small open subsets of $X \backslash K$.

For $R>0$, let $X^{R}$ be an arbitrary union of finitely many connected components of the set $H^{-1}\left(B_{n}(R)\right) \subset X$, and let $Z^{R}=H\left(X^{R}\right)=B_{n}(R)$. Note that the map $H: X^{R} \rightarrow B(R)$ is proper. Let $\left\{X^{k}\right\}$ be a normal exhaustion of $X$ and let the set $U_{k}$ be an open Stein neighborhood of $X^{k}$ contained in $X^{k+1}$, for each $k$. By the above definition the set $X^{k}$ is Runge in $X^{k+1}$. We may assume that $S \cap\left(\partial X^{k} \cup \partial U_{k}\right)=\varnothing$. By $\psi(z)=\|z\|^{2}$ we denote the square of euclidean norm on $\mathbb{C}^{n}$.

We will construct a sequence of maps $G_{k}: U_{k} \rightarrow \mathbb{C}^{N-n}$ and a decreasing sequence $\varepsilon_{j} \rightarrow 0$ such that

(i) $\left(H, G_{k}\right): U_{k} \rightarrow \mathbb{C}^{N}$ is weakly regular and injective,

(ii) $\left\|G_{k}-G_{k-1}\right\|_{X^{k-1}}<2^{-k} \varepsilon_{k-1}$,

(iii) if $G^{\prime}: U_{k} \rightarrow \mathbb{C}^{N-n}$ satisfies $\left\|G^{\prime}-G_{k}\right\|_{X^{k}}<\varepsilon_{k}$ then $\left(H, G^{\prime}\right): X \rightarrow \mathbb{C}^{N}$ is weakly regular and injective,

(iv) $\inf \left\{\left\|G_{k}(x)\right\|: x \in\left(H^{-1}\left(B_{n}(k-1)\right) \backslash X^{k-1}\right) \cap X^{k}\right\}>k-1$,

(v) $D G_{k}\left(s_{j}\right)=D\left(\Phi_{n+1}, \ldots, \Phi_{N}\right)\left(s_{j}\right)$ for all $j$ and $k$, whenever the expression makes sense.

Note that the sequence $G_{k}$ converges uniformly on compact sets to a map $G$ such that the map $(H, G): X \rightarrow \mathbb{C}^{N}$ is weakly regular and injective by (i), (ii) and (iii), and proper by (iv). Condition (v) could be omitted since weak regularity is stable under small perturbations; in fact the construction ensures that we get $(\mathrm{v})$ for free. 
Before proceeding to the construction of maps the $G_{k}$, we define stratifications of $X$ and $\mathbb{C}^{n}$ which we will need in the sequel.

Lemma 3.5 [Schürmann 1992; Prezelj 2003]. There exist stratifications $X_{n}:=$ $X^{R} \supset X_{n-1} \supset \cdots \supset X_{0} \supset X_{-1}=\varnothing$ and $Z_{n}:=Z^{R} \supset Z_{n-1} \supset \cdots \supset Z_{0} \supset Z_{-1}=\varnothing$, with $X_{0}, Z_{0} \neq \varnothing$, such that

(i) $X_{0} \supset X^{R} \cap S$ and $Z_{0} \supset H\left(S \cap X^{R}\right)$,

(ii) $X_{j}=H^{-1}\left(Z_{j}\right) \cap X^{R}$,

(iii) the sets $X_{j}$ and $Z_{j}$ have dimension at most $j$ and the sets $X_{j}^{*}:=X_{j} \backslash X_{j-1}$ and $Z_{j}^{*}=Z_{j} \backslash Z_{j-1}$ are either complex $j$-dimensional manifolds or empty,

(iv) if $X_{j}^{*}$ is not empty, the map $H: X_{j}^{*} \rightarrow Z_{j}^{*}$ is an immersion for $j \in\{0, \ldots, n\}$,

(v) the rank of $H$ is constant on each connected component of the set $X_{j}^{*}$ for each $j \in\{0, \ldots, n\}$.

We quote some more results from [Schürmann 1992] (the almost proper map $H$ is fixed). The original theorems deal with immersions and injective immersions; in our case the term "immersion" will be replaced with the term "weakly regular". Let $q=N-n$, fix some $R>0$ and let $\left\{X_{j}\right\}$ and $\left\{Z_{j}\right\}$ be the stratifications from Lemma 3.5.

Theorem 3.6. Choose $j \in\{1, \ldots, n\}$ and $r, r_{1}, r_{2} \in \mathbb{R} \backslash\{0\}$ such that $r_{2}>0$ and $r_{1}<r_{2}<r<R$. Let $r_{1}^{2}$ and $r_{2}^{2}$ be regular values for $\left.\psi\right|_{Z_{j}^{*}}$ and suppose that $\left(X_{j-1} \cap \overline{X^{r_{2}}}\right) \cup\left(X_{j} \cap \overline{X^{r}}\right)$ is not empty. Let $f: X^{r} \rightarrow \mathbb{C}^{q}$ be a holomorphic map such that the map $(H, f): X^{r} \rightarrow \mathbb{C}^{N}$ is weakly regular on $\left(X_{j-1} \cap \overline{X^{r}}\right) \cup\left(X_{j} \cap \overline{X^{r_{1}}}\right)$. Then:

(i) If $r_{1}<0$, there is a holomorphic map $f^{\prime}: X^{r_{2}} \rightarrow \mathbb{C}^{q}$ such that $f^{\prime}-\left.f\right|_{X^{r_{2}}}$ is in $\Gamma\left(X^{r_{2}}, \mathscr{g}\left(X_{j-1}\right)^{2} \cap \mathscr{g}\left(X_{j}\right)\right)^{q}$ and $\left(H, f^{\prime}\right)$ is weakly regular on $X_{j} \cap X^{r_{2}}$.

(ii) If $r_{1}>0$ then $f$ can be approximated arbitrarily well on $X^{r_{1}}$ by a holomorphic map $f^{\prime}: X^{r_{2}} \rightarrow \mathbb{C}^{q}$ such that $f^{\prime}-\left.f\right|_{X^{r_{2}}}$ is in $\Gamma\left(X^{r_{2}}, \mathscr{g}\left(X_{j-1}\right)^{2} \cap \mathscr{g}\left(X_{j}\right)\right)^{q}$ and $\left(H, f^{\prime}\right)$ is weakly regular on $X_{j} \cap X^{r_{2}}$.

Proof. Since the sheaf $\mathscr{f}\left(X_{j-1}\right)^{2} \cap \mathscr{g}\left(X_{j}\right)$ is coherent, there are finitely many holomorphic sections

$$
f_{1}, \ldots, f_{M} \in \Gamma\left(X, \mathscr{g}\left(X_{j-1}\right)^{2} \cap \mathscr{g}\left(X_{j}\right)\right)^{q}
$$

which generate $\Gamma\left(X^{r_{2}}, \mathscr{f}\left(X_{j-1}\right)^{2} \cap \mathscr{g}\left(X_{j}\right)\right)^{q}$. We seek a map $f^{\prime}$ of the form

$$
f^{\prime}=f+\sum \alpha_{j} f_{j}
$$

where $\alpha=\left(\alpha_{1}, \ldots, \alpha_{m}\right)$ is regarded as a section of the trivial bundle $X^{r} \times \mathbb{C}^{M}$. Denote by $K=\operatorname{Ker} D H \subset T X$ the kernel of $D H$ and note that the definition of $X_{j}^{*}$ implies that $\left.K\right|_{X_{j}^{*}}$ is a vector bundle. Let $\Sigma \subset\left(X_{j} \cap X^{r}\right) \times \mathbb{C}^{M}=: V$ be the set of all 
$\left(x, a_{1}, \ldots, a_{M}\right)$ such that the map $\left(H, f+\sum a_{j} f_{j}\right)$ is not weakly regular in $x$. Let $p: V \rightarrow\left(X_{j} \cap X^{r}\right)$ be the trivial projection. Then because $(H, f)$ is weakly regular on $X_{j-1} \cap X^{r}$ and the maps $f_{j}$ vanish to second order on $X_{j-1}$, the set $\Sigma$ is a subset of $X_{j}^{*} \times \mathbb{C}^{M}$. Since $\left.K\right|_{X_{j}^{*}}$ is a vector bundle, $\Sigma$ is the set of all $\left(x, a_{1}, \ldots, a_{M}\right)$ in $X_{j}^{*} \times \mathbb{C}^{M}$ such that the map $K_{x} \rightarrow \mathbb{C}^{q^{\prime}}$ given by $v \mapsto D f(x) v+\sum a_{i} D f_{j}(x) v$, is not injective, so $\Sigma$ is analytic in $X_{j}^{*} \times \mathbb{C}^{M}$. But we want $\Sigma$ to be analytic in $V$ which means we have to prove that $\Sigma$ is closed in $V$. Now, since $(H, f)$ is weakly regular on $X_{j-1} \cap X^{r}$ any map of the form $\left(H, f+\sum \alpha_{j} f_{j}\right)$ is weakly regular on the same set and because weak regularity is an open condition, the map $\left(H, f+\sum \alpha_{j} f_{j}\right)$ is also weakly regular in some neighborhood of $X_{j-1} \cap X^{r}$ which means that $\Sigma$ is a closed in $V$. As in [Schürmann 1992] we prove that the projection $p: V \backslash \Sigma \rightarrow\left(X_{j}^{*} \cap X^{r}\right)$ is a locally trivial fibration which admits a spray.

Our goal is to find a holomorphic section $\alpha$ of $\left(X^{r} \times \mathbb{C}^{M}\right) \backslash \Sigma \rightarrow X^{r}$. The zero section on $\left(X_{j-1} \cap \overline{X^{r}}\right) \cup\left(X_{j} \cap \overline{X^{r_{1}}}\right)$ is a section of

$$
\left(\left(X^{r} \cap X_{j}\right) \times \mathbb{C}^{M}\right) \backslash \Sigma \rightarrow\left(X^{r} \cap X_{j}\right)
$$

because the map $(H, f)$ is weakly regular on $\left(X_{j-1} \cap \overline{X^{r}}\right) \cup\left(X_{j} \cap \overline{X^{r_{1}}}\right)$. And since weak regularity is an open condition, the zero section defined in a neighborhood of the set $\left(X_{j-1} \cap \overline{X^{r}}\right) \cup\left(X_{j} \cap \overline{X^{r_{1}}}\right)$ is a section of $\left(\left(X^{r} \cap X_{j}\right) \times \mathbb{C}^{M}\right) \backslash \Sigma \rightarrow\left(X^{r} \cap X_{j}\right)$ as well. As in [Schürmann 1992] this section can be extended to a continuous section of $\left(\left(X^{r} \cap X_{j}\right) \times \mathbb{C}^{M}\right) \backslash \Sigma \rightarrow\left(X^{r} \cap X_{j}\right)$. Then the h-principle applies (if $r_{1}<0$ we use the existence version and if $r_{1}>0$ the approximation version) which yields a holomorphic section $\alpha^{\prime}$ of $\left(\left(X^{r} \cap X_{j}\right) \times \mathbb{C}^{M}\right) \backslash \Sigma \rightarrow\left(X^{r} \cap X_{j}\right)$. This section can be trivially extended to a holomorphic section $\alpha$ of $\left(X^{r} \times \mathbb{C}^{M}\right) \backslash \Sigma \rightarrow X^{r}$.

Remark 3.7. The maps $f_{j}$ used in Theorem 3.6 vanished to the first order on $X_{j}$, which means that if the initial map $f$ is such that $(H, f)$ is injective on $X_{j} \cap X^{r}$ then the map $\left(H, f^{\prime}\right)$ is also injective on $X_{j} \cap X^{r}$.

Theorem 3.8. Choose $j \in\{1, \ldots, n\}$ and $r, r_{1}, r_{2} \in \mathbb{R} \backslash\{0\}$ such that $r_{2}>0$ and $r_{1}<r_{2}<r<R$. Let $r_{1}^{2}$ and $r_{2}^{2}$ be regular values for $\left.\psi\right|_{Z_{j}^{*}}$ and suppose that $\left(X_{j-1} \cap \overline{X^{r_{2}}}\right) \cup\left(X_{j} \cap \overline{X^{r}}\right)$ is not empty. Let $f: X^{r} \rightarrow \mathbb{C}^{q}$ be a holomorphic map such that the map $(H, f): X^{r} \rightarrow \mathbb{C}^{N}$ is injective and weakly regular on $\left(X_{j-1} \cap \overline{X^{r}}\right) \cup\left(X_{j} \cap \overline{X^{r_{1}}}\right)$. Then:

(i) If $r_{1}<0$ there is a holomorphic map $f^{\prime}: X^{r_{2}} \rightarrow \mathbb{C}^{q}$ such that $f^{\prime}-\left.f\right|_{X^{r_{2}}}$ is in $\Gamma\left(X^{r_{2}}, \mathscr{F}\left(X_{j-1}\right)^{2}\right)^{q}$ and such that $\left(H, f^{\prime}\right)$ is injective and weakly regular on $X_{j} \cap X^{r_{2}}$.

(ii) If $r_{1}>0$ the map $f$ can be approximated arbitrarily well on the set $X^{r_{1}}$ by a holomorphic map $f^{\prime}: X^{r_{2}} \rightarrow \mathbb{C}^{q}$ such that $f^{\prime}-\left.f\right|_{X^{r_{2}}} \in \Gamma\left(X^{r_{2}}, \mathscr{g}\left(X_{j-1}\right)^{2}\right)^{q}$ and $\left(H, f^{\prime}\right)$ is injective and weakly regular on $X_{j} \cap X^{r_{2}}$. 
Proof. Since the sheaves $\mathscr{F}=H_{*} \mathcal{O}\left(X^{R}\right)$ and $\mathscr{f}\left(Z_{j-1}\right)^{2} \mathscr{F}$ are coherent, there exist finitely many holomorphic sections $\psi_{1}, \ldots \psi_{M} \in \Gamma\left(Z, \mathscr{g}\left(Z_{j-1}\right)^{2} \mathscr{F}^{q}\right)^{q}$ generating $\Gamma\left(Z^{r_{2}}, \mathscr{g}\left(Z_{j-1}\right)^{2} \mathscr{F}\right)^{q}$. Let $f_{j} \in \Gamma\left(X^{r}, \mathcal{O}(X)\right)^{q}$ be liftings of the sections $\psi_{j}$. We are looking for the map $f^{\prime}$ of the form

$$
f^{\prime}=f+\sum\left(\alpha_{j} \circ H\right) \cdot f_{j},
$$

where $\alpha=\left(\alpha_{1}, \ldots, \alpha_{m}\right)$ is regarded as a section of the trivial bundle $B(r) \times \mathbb{C}^{M}$. Let $\Sigma \subset\left(Z_{j} \cap B(r)\right) \times \mathbb{C}^{M}=: V$ be the set of all $\left(z, a_{1}, \ldots, a_{M}\right)$, such that the map $H^{-1}(z) \rightarrow \mathbb{C}^{n}$, given by $x \mapsto f(x)+\sum a_{j} f_{j}(x)$, is not injective. Let the map $p: V \rightarrow\left(Z_{j} \cap B(r)\right)$ be the trivial projection. Then because $(H, f)$ is injective on $\left(X_{j-1} \cap \overline{X^{r}}\right) \cup\left(X_{j} \cap \overline{X^{r_{1}}}\right)$ and the maps $f_{j}$ vanish to the second order on $X_{j-1}$, the set $\Sigma$ is an analytic subset of $Z_{j}^{*} \times \mathbb{C}^{M}$. As above we want $\Sigma$ to be an analytic subset of $V$, that is, closed in $V$. Since $(H, f)$ is injective and weakly regular on $X_{j-1} \cap X^{r}$ and the maps $f_{j}$ vanish to the second order on $X_{j-1}$, any map of the form $\left(H, f+\sum\left(\alpha_{j} \circ H\right) \cdot f_{j}\right)$ is injective and weakly regular on the same set and, because being injective and weakly regular is an open condition, such map is also injective and weakly regular in some neighborhood of $X_{j-1} \cap X^{r}$. This means that the set $\Sigma$ is closed in $V$. As in [Schürmann 1992] we prove that $p: V \backslash \Sigma \rightarrow\left(Z_{j}^{*} \cap B(r)\right)$ is a locally trivial fibration which admits a spray.

We seek a holomorphic section $\alpha$ of the submersion $\left(B(r) \times \mathbb{C}^{M}\right) \backslash \Sigma \rightarrow B(r)$. The zero section defined on $\left(Z_{j-1} \cap \bar{B}(r)\right) \cup\left(Z_{j} \cap \bar{B}\left(r_{1}\right)\right)$ is a section of the map $\left(\left(B(r) \cap Z_{j}\right) \times \mathbb{C}^{M}\right) \backslash \Sigma \rightarrow\left(B(r) \cap Z_{j}\right)$ because the map $(H, f)$ is weakly regular and injective on $\left(X_{j-1} \cap \overline{X^{r}}\right) \cup\left(X_{j} \cap \overline{X^{r_{1}}}\right)$. And since being injective and weakly regular is an open condition, the zero section defined in a neighborhood of the set $\left(Z_{j-1} \cap \bar{B}(r)\right) \cup\left(Z_{j} \cap \bar{B}\left(r_{1}\right)\right)$ is a section of $\left(\left(B(r) \cap Z_{j}\right) \times \mathbb{C}^{M}\right) \backslash \Sigma \rightarrow\left(B(r) \cap Z_{j}\right)$ as well. As in [Schürmann 1992] this section can be extended to a continuous section of $\left(\left(B(r) \cap Z_{j}\right) \times \mathbb{C}^{M}\right) \backslash \Sigma \rightarrow\left(B(r) \cap Z_{j}\right)$. Then the h-principle applies (if $r_{1}<0$ we use the existence version and if $r_{1}>0$ the approximation version) and yields a holomorphic section $\alpha^{\prime}$ of $\left(\left(B(r) \cap Z_{j}\right) \times \mathbb{C}^{M}\right) \backslash \Sigma \rightarrow\left(B(r) \cap Z_{j}\right)$, which can be trivially extended to a holomorphic section $\alpha$ of $\left(B(r) \times \mathbb{C}^{M}\right) \backslash \Sigma \rightarrow B(r)$.

At the beginning of this section we defined the map $\Phi$. Now we define

$$
f=\left(\Phi_{n+1}, \ldots, \Phi_{N}\right) .
$$

The map $(H, f)$ clearly is weakly regular on $S$ and injective in a small neighborhood of $S$, since $\Phi_{N}\left(s_{j}\right)=j$. Using in turn Theorem 3.8 and Theorem 3.6 we can proceed by induction over the strata starting with $X_{0}$ (as in [Schürmann 1992]), and using the fact that being weakly regular or injective and weakly regular is an open condition, to obtain the following results: 
Theorem 3.9 (Existence). Let $R>0$ and let $X^{R}$ be the union of a finite number of connected components of the set $H^{-1}\left(B_{n}(R)\right)$. For $r \in(0, R)$ let $X^{r}:=$ $X^{R} \cap H^{-1}\left(B_{n}(r)\right)$. There exists a holomorphic map $G: X^{r} \rightarrow \mathbb{C}^{q}$ satisfying the conditions

$\alpha(r):$ the map $(H, G): X^{r} \rightarrow \mathbb{C}^{N}$ is injective and weakly regular, and $\beta(r):(H, G)$ coincides with $\Phi$ to the second order $S \cap X^{r}$.

Theorem 3.10 (Approximation). Let $R, r>0, X^{R}$ and $X^{r}$ be as in Theorem 3.9 Choose $r_{1} \in(r, R)$ and set $X^{r_{1}}:=X^{R} \cap H^{-1}\left(B_{n}\left(r_{1}\right)\right)$. If a holomorphic map $G: X^{r} \rightarrow \mathbb{C}^{q}$ satisfies conditions $\alpha(r)$ and $\beta(r)$ from Theorem 3.9, it can be approximated arbitrarily well on the set $X^{r}$ by a map $G^{\prime}: X^{r_{1}} \rightarrow \mathbb{C}^{q}$ satisfying $\alpha\left(r_{1}\right)$ and $\beta\left(r_{1}\right)$ from Theorem 3.9.

Remark 3.11. Note that the induction preserves the derivatives of $\Phi$ at the points in $S$ since they are contained in $X_{0}$ and the maps $f_{j}$ in Theorem 3.6 and Theorem 3.8 vanish to second order on $X_{0}$.

Proof of the main theorem. Now we can construct the maps $G_{j}$ and the sequence $\varepsilon_{j} \rightarrow 0$ with the required properties (i)-(v). First we consider the case $k=1$. By the existence Theorem 3.9 there is a map $G_{1}: X^{1} \rightarrow \mathbb{C}^{q}$ with properties (i) and (v). By Lemma 2.13 there is an $\varepsilon_{1}>0$ such that (iii) holds. Now we prove the induction step. Assume that $G_{1}, \ldots, G_{k}$ and $\varepsilon_{1}, \ldots, \varepsilon_{k}$ have already been constructed. Let $X^{k^{\prime}}:=X^{k+1} \cap\left(H^{-1}\left(B_{k}\right) \backslash X^{k}\right)$, that is, $X^{k^{\prime}}$ is the union of those connected components of $H^{-1}(B(k))$ which lie in $X^{k+1}$ but not in $X^{k}$. By Theorem 3.9 there is a map $G_{k}^{\prime}$ satisfying (i). By adding a sufficiently large positive constant we may assume that $\left\|G^{\prime}\right\|_{X^{k^{\prime}}}>2 k$ and that the map $G^{\prime}: X^{k} \cup X^{k^{\prime}} \rightarrow \mathbb{C}^{q}$, defined by $\left.G^{\prime}\right|_{X^{k}}=G_{k}, G_{X^{k^{\prime}}}^{\prime}=G_{k}^{\prime}$ is such that $\left(H, G^{\prime}\right): X^{k} \cup X^{k^{\prime}} \rightarrow \mathbb{C}^{N}$ is injective. Now the assumptions of Theorem 3.9 are fulfilled so there exists a map $G_{k+1}: X^{k+1} \rightarrow \mathbb{C}^{N}$ satisfying (i), (ii), (iv) and (v). As above, there exists $\varepsilon_{k+1} \in\left(0, \varepsilon_{k}\right)$ such that (ii) holds as well.

\section{References}

[Acquistapace et al. 1975] F. Acquistapace, F. Broglia, and A. Tognoli, "A relative embedding theorem for Stein spaces”, Ann. Scuola Norm. Sup. Pisa Cl. Sci. (4) 2:4 (1975), 507-522. MR 53 \#870 Zbl 0313.32020

[Chirka 1989] E. M. Chirka, Complex analytic sets, Mathematics and its Applications 46, Kluwer, Dordrecht, 1989. MR 92b:32016 Zbl 0683.32002

[Forstnerič and Prezelj 2001] F. Forstnerič and J. Prezelj, "Extending holomorphic sections from complex subvarieties”, Math. Z. 236:1 (2001), 43-68. MR 2002b:32017 Zbl 0968.32005

[Gromov 1989] M. Gromov, "Oka's principle for holomorphic sections of elliptic bundles", J. Amer. Math. Soc. 2:4 (1989), 851-897. MR 90g:32017 Zbl 0686.32012 
[Ohsawa 1984a] T. Ohsawa, "Holomorphic embedding of compact s.p.c. manifolds into complex manifolds as real hypersurfaces", pp. 64-76 in Differential geometry of submanifolds (Kyoto, 1984), edited by K. Kenmotsu, Lecture Notes in Math. 1090, Springer, Berlin, 1984. MR 86j:32047 Zbl 0578.32032

[Ohsawa 1984b] T. Ohsawa, "Global realization of strongly pseudoconvex CR manifolds", Publ. Res. Inst. Math. Sci. 20:3 (1984), 599-605. MR 85m:32013 Zbl 0568.32014

[Prezelj 2003] J. Prezelj, "Interpolation of embeddings of Stein manifolds on discrete sets", Math. Ann. 326:2 (2003), 275-296. MR 2004b:32043 Zbl 1037.32024

[Rossi 1965] H. Rossi, "Attaching analytic spaces to an analytic space along a pseudoconcave boundary", pp. 242-256 in Proc. Conf. Complex Analysis (Minneapolis, 1964), edited by A. Aeppli et al., Springer, Berlin, 1965. MR 31 \#381 Zbl 0143.30301

[Schürmann 1992] J. Schürmann, Einbettungen Steinscher Räume in affine Räume minimaler Dimension, Schriftenreihe des Mathematischen Instituts (3. Serie) 7, Universität Münster, Münster, 1992. MR 94g:32012 Zbl 0766.32014

[Schürmann 1997] J. Schürmann, "Embeddings of Stein spaces into affine spaces of minimal dimension”, Math. Ann. 307:3 (1997), 381-399. MR 98a:32011 Zbl 0881.32007

[Stutz 1972] J. Stutz, "Analytic sets as branched coverings", Trans. Amer. Math. Soc. 166 (1972), 241-259. MR 48 \#2420 Zbl 0239.32006

Received November 21, 2001. Revised February 28, 2004.

\author{
JASNA PREZELJ \\ DEPARTMENT OF MATHEMATICS \\ UNIVERSITY OF LJUBLJANA \\ JADRANSKA 19 \\ SI-1000 LJUBLJANA \\ SLOVENIA \\ jasna.prezelj@fmf.uni-lj.si
}

